\title{
NEK5 Activity Regulates the Mesenchymal and Migratory Phenotype in Breast Cancer Cells
}

\section{Margarite Matossian ( $\nabla$ mmatossi@tulane.edu )}

Tulane University School of Medicine https://orcid.org/0000-0002-9527-1043

\section{Steven Elliott}

Tulane University School of Medicine

\section{Van T. Hoang}

Tulane University School of Medicine

Hope E. Burks

Tulane University School of Medicine

\section{Maryl K. Wright}

Tulane University School of Medicine

Madlin Alzoubi

Tulane University School of Medicine

Thomas Yan

Tulane University School of Medicine

\section{Tiffany Chang}

Tulane University School of Medicine

Henri Wathieu

Tulane University School of Medicine

\section{Gabrielle Windsor}

Tulane University School of Medicine

\section{Alifiani Bo Hartono}

Tulane University School of Medicine

\section{Sean Lee}

Tulane University School of Medicine

\section{William J. Zuercher}

University of North Carolina at Chapel Hill Eshelman School of Pharmacy

\section{David H. Drewry}

University of North Carolina Lineberger Comprehensive Cancer Center

\section{Carrow Wells}

University of North Carolina at Chapel Hill Eshelman School of Pharmacy

Nirav Kapadia

University of North Carolina at Chapel Hill Eshelman School of Pharmacy Aaron Buechlein 
Indiana University School of Medicine

\section{Fang Fang}

Indiana University School of Medicine

\section{Kenneth P. Nephew}

Indiana University School of Medicine

\section{Bridgette M. Collins-Burow}

Tulane University School of Medicine

\section{Matthew E. Burow}

Tulane University School of Medicine

\section{Research Article}

Keywords: breast cancer, never-in-mitosis A-related kinase, kinase target, metastasis, cell motility, mesenchymal phenotype

Posted Date: April 26th, 2021

DOI: https://doi.org/10.21203/rs.3.rs-412293/v1

License: (1) (1) This work is licensed under a Creative Commons Attribution 4.0 International License. Read Full License 


\section{Abstract}

Purpose: Breast cancer remains a prominent global disease affecting women worldwide despite the emergence of novel therapeutic regimens. Metastasis is responsible for most cancer-related deaths, and acquisition of a mesenchymal and migratory cancer cell phenotypes contributes to this devastating disease. The utilization of kinase targets in drug discovery have revolutionized the field of cancer research but despite impressive advancements in kinase-targeting drugs, a large portion of the human kinome remains under-studied in cancer. NEK5, a member of the Never-in-mitosis kinase family, is an example of such an understudied kinase. Here, we characterized the function of NEK5 in breast cancer.

Methods: Stably overexpressing NEK5 cell lines (MCF-7) and shRNA knockdown cell lines (MDA-MB-231, TU-BcX-4IC) were utilized. Cell morphology changes were evaluated using immunofluorescence and quantification of cytoskeletal components. Cell proliferation was assessed by Ki-67 staining and transwell migration assays tested cell migration capabilities. In vivo experiments with murine models were necessary to demonstrate NEK5 function in breast cancer tumor growth and metastasis.

Results: NEK5 activation altered breast cancer cell morphology and promoted cell migration independent of effects on cell proliferation. NEK5 overexpression or knockdown does not alter tumor growth kinetics but promotes or suppresses metastatic potential in a cell type specific manner, respectively.

Conclusion: While NEK5 activity modulated cytoskeletal changes and cell motility, NEK5 activity affected cell seeding capabilities but not metastatic colonization or proliferation in vivo. Here we characterized NEK5 function in breast cancer systems and we implicate NEK5 in regulating specific steps of metastatic progression.

\section{Background}

Breast cancer remains a predominant malignancy worldwide. In the United States, there are more than 3.8 million women living with a history of breast cancer, including over 150,000 women living with metastatic disease [1]. With profound advancements in breast cancer therapeutic management, mortality rates have decreased overall. However, invasive breast cancer remains difficult to manage with current regimens; approximately $13 \%$ of women will be diagnosed with invasive breast cancer in their lifetime [1]. Classically, breast cancers (BC) were categorized based on the receptors they expressed, estrogen receptor positive (ER+), progesterone receptor positive (PR+), HER2/Neu amplified, or triple negative breast cancer (TNBC) [2, 3]. More recently, Lehmann et al. introduced a new classification system for breast cancers based on gene expression profiling of the tumors: Basal-like 1, basal-like 2, luminal androgen receptor and mesenchymal $[4,5]$. One of the underlying features that determines the clinical behavior of a tumor is the propensity of cancer cells to migrate and invade in distal tissue sites [6-8]. In addition to the complex and heterogenous nature of breast cancers, metastasis and tumor recurrence are obstacles in effectively treating breast cancer [9-11]. There is an urgent need to continue to characterize 
potential drivers of these processes to formulate effective targeted therapeutic regimens for breast cancer.

Kinase-targeting agents are widely used in anti-cancer therapeutic regimens. Kinases are enzymes that modulate cell signaling pathways responsible for a range of processes including cell proliferation, survival, motility, and apoptosis. Out of the 538 known human kinases, many have key roles in carcinogenesis and metastasis of various cancer types $[12,13]$. Kinase inhibitors account for approximately $25 \%$ of all currently investigated therapeutics [14]. Currently, several kinase targets are being pursued in difficult-to-treat breast cancer types, such as triple negative breast cancers lacking commonly targeted receptors HER2/Neu and estrogen receptor, including cyclin-dependent kinases (CDKs), BRAF, lipid kinases like phosphatidylinositol 3-kinase (PI3K) alpha, AKT, CHK1 and epidermal growth factor receptor (EGFR) $[14,15]$. However, because the human kinome is vast there exist many unexplored targets in cancer [16].

Kinases that regulate key processes in cell survival and division, such as mitosis, are important to study as novel targets in cancer due to frequent upregulation in human cancer [17-19]. The three major families of mitotic kinases are: Polo, Aurora, and the Never-in-mitosis (NEK) kinase families [20, 21]. Of these kinases, the NEK members remain the least studied. NEK family members regulate specific mitotic events, including centrosome separation, spindle assembly and cytokinesis [21, 22]. While many of the eleven members of the NEK family have been extensively characterized, NEK5 function remains widely understudied. Various groups have shown that NEK5 expression is upregulated in specific tumor types (colon, lung, thyroid, breast) compared to normal cells/tissue [23]. NEK5 has the highest endogenous protein expression in testes, and it has been proposed to regulate cilia motility [24]. To support this, NEK5 expression is higher in ciliated tissues, including colon and lung [23].

Recent studies demonstrate a role for NEK5 in regulation of microtubule assembly and activity during mitosis [25]. Because microtubule activation is associated with enhanced cell migration and motility in cancer systems, these findings led us to interrogate a possible role for NEK5 in breast cancer cell migration. Furthermore, NEK5 has been shown to have an integral role in the DNA damage response, through interactions with topoisomerase $\| \beta$ [26]. Recently, Pei et al. evaluated NEK5 mRNA and protein expression in breast cancer. Upregulation of NEK5 expression in breast cancer cells promoted tumor progression and silencing of NEK5 suppressed proliferation and inhibited migration and invasion. This important study demonstrated an integral role for NEK5 in cell proliferation and migration pathways in breast cancer through regulation of Cyclin A2 function [27].

In this study, we provide further evidence that supports a potential role for NEK5 in driving breast cancer cell motility and we parse out specific processes in breast cancer biology that are regulated, or not regulated, by NEK5 activity. We employed iterations of patient-derived xenograft (PDX) models as physiologically relevant tools in our project to provide more translational evidence of our work. Given the understudied status of NEK5, and the recent interest in further characterization of the NEK family in 
cancer biology, our work is crucial to elucidate specific roles for NEK5 in breast cancer to evaluate its potential as a therapeutic target.

\section{Methods}

\section{Cell culture and reagents}

Breast cancer cell lines MDA-MB-231, andMCF-7 were acquired from American Type Culture Collection (ATCC). Liquid nitrogen stocks were generated when cells were received, and cells were maintained in culture until the start of each study. Cells were maintained in DMEM supplemented with $10 \%$ FBS, nonessential amino acids (NEAA), MEM amino acids, anti-anti $(100 \mathrm{U} / \mathrm{mL})$, sodium pyruvate and porcine insulin $\left(1 \times 10^{-10} \mathrm{~mol} / \mathrm{L}\right)$ at $37^{\circ} \mathrm{C}$ in humidified $5 \% \mathrm{CO}_{2}$. Dulbecco's modified Eagle's medium (DMEM), Dulbecco's phosphate-buffered saline (DPBS), phenol-red free DMEM, fetal bovine serum (FBS), minimal essential amino acids (MEMAA), non-essential amino acids (NEAA), antibiotic/anti-mitotic penicillin/streptomycin (pen/strep), sodium pyruvate, L-glutamine, trypsin/EDTA, trypan blue stain (0.4\%) and ethylenediaminetetraacetic acid (EDTA 0.5 M, pH 8) were obtained from GIBCO (Invitrogen; Carlsbad $\mathrm{CA}$ ). Insulin was purchased from Sigma-Aldrich (St. Louis MO). For select experiments, cells were grown in phenol red-free DMEM supplemented with $5 \%$ charcoal-stripped FBS (HyClone, Thermo Scientific, Logan UT) and with NEAA, MEM amino acids, Gluta-Max and penicillin (100 U/mL) for 48 hours.

\section{Generation of PDX-derived primary cell lines}

To generate cell lines derived from patient tumors, after resection small $\left(2 \times 2 \mathrm{~mm}^{2}\right)$ explants of the original TU-BCX-4IC tumor were plated in adherent culture conditions in a 6-well plate with DMEM supplemented with $10 \%$ FBS. Cells grew out from the explants for 2-3 weeks; media was changed every 72 hours. When cell populations were observed, single populations were cloned into 96-well plates and expanded, and a cell line was established from one of the clones [28].

\section{Generation of stably overexpressing cell lines}

Plasmids for overexpression experiments were purchased from Addgene (Watertown, MA). MCF7 cells were plated in $10 \mathrm{~cm}$ dishes and cells adhered overnight at $37^{\circ} \mathrm{C}$ in $5 \% \mathrm{CO}_{2}$. Cells were transfected with 5 $\mu \mathrm{g}$ of plasmid in $300 \mu \mathrm{L}$ Opti-MEM. Transfection was accomplished using $15 \mu \mathrm{L}$ Attractene per manufacturer's instructions (Qiagen, Valencia, CA). Media was changed the following day and cells were treated with gradually increasing amounts of the selectable marker neomycin every two days. Once stable cells were obtained, viable colonies were cloned or pooled. Stable overexpression was confirmed by qPCR and Western blot.

\section{Generation of stable knockdown cell lines}

A doxycycline-inducible shRNA-system kindly provided by Dr. Sean Lee (Tulane University Department of Pathology) was utilized for knockdown experiments. The shLT3GEPIR empty vector was utilized as the backbone and was modified to remove the SAPI sequence to increase transfection efficiency. MDA-MB- 
231 or TU-BcX-4IC cells were plated in $10 \mathrm{~cm}$ dishes and cells adhered overnight at $37^{\circ} \mathrm{C}$ in $5 \% \mathrm{CO}_{2}$. Cells were transfected with $5 \mu \mathrm{g}$ of plasmid in $300 \mu \mathrm{L}$ Opti-MEM. Transfection was accomplished using $15 \mu \mathrm{L}$ Attractene per manufacturer's instructions (Qiagen, Valencia, CA). Media was changed the following day and cells were treated with gradually increasing amounts of the selectable marker puromycin every two days. To induce knockdown, cells were treated with doxycycline $(2 \mu \mathrm{g} / \mathrm{mL})$ or grown in the absence of doxycycline as a negative control for 48 hours. Stable knockdown was confirmed by qPCR and Western blot.

\section{Crystal violet staining}

Cells were plated at 5,000 cells per well in 96 -well TC plate in $5 \%$ charcoal stripped (CS) DMEM. The cells incubated for 48 hours in CS DMEM media at $37^{\circ} \mathrm{C}$ with $5 \% \mathrm{CO}_{2}$. Cells were fixed with gluteraldehyde (10 $\mu \mathrm{L})$ for 20 minutes. After rinsing and drying the plate, cells were stained with $50 \mu \mathrm{M} 0.1 \%$ crystal violet in $90 \%$ methanol for 20 minutes. After another rinse, the cells were left overnight to dry, and the following day stained cells were visualized with an inverted microscope and images were recorded.

\section{Transwell migration assay}

Migration assays were performed following the manufacturer's instructions (BD Biosciences, San Jose, CA, USA). MDA-MB-231 cells were exposed to CS DMEM ( $5 \%$ FBS) for 48 hours. Transwell inserts ( $8 \mu \mathrm{M}$; BD biosciences; San Jose, CA) were placed into each well containing $1 \mathrm{~mL}$ 10\% FBS DMEM media. Pretreated cells seeded in opti-MEM suspension were added to the top of each insert ( $500 \mu \mathrm{L}, 25,000$ cells per well). After 24 hours, membranes were scrubbed with a cotton tip to remove non-migrated cells and membranes were fixed in formalin (10\%) stained with crystal violet, removed, and mounted on glass slides. Migrated cells were visualized by microscopy and counted. Data is represented as number of migrated cells per field of view for triplicate experiments.

\section{Crystal violet staining}

Cells were plated at 5,000 cells per well in 96 -well plate in $5 \%$ charcoal stripped FBS (CS-FBS) and DMEM media. After 48 hours the plate was then harvested by adding $10 \mu \mathrm{L}$ glutaraldehyde to each well for 20 minutes. After rinsing and drying the plate, the cells were stained with $50 \mu \mathrm{L} 0.1 \%$ crystal violet in UltraPur water (Invitrogen, Cat. No. 10977-023) for 20 minutes. After another rinse, the cells were left overnight to dry, and the following day cell morphology was visualized with brightfield microscopy using an inverted microscope (Nikon Microscope) and images were recorded. Cells were lysed with $33 \%$ acetic acid and absorbance was measured at $570 \mathrm{nM}$ to quantify data and evaluate relative cell viability.

\section{RNA isolation and quantitative real time PCR}

Cells were harvested, and total RNA was isolated using the Quick RNA Mini Prep Kit in accordance with the manufacturer's protocol (Zymo Research, Irvine, CA) following manufacturer's protocol. The concentration and quality of RNA was determined by absorbance $(260,280 \mathrm{~nm})$ using the NanoDrop ND1000. Total RNA $(1 \mu \mathrm{g})$ was reverse-transcribed qScript cDNA synthesis kit (Quantabio, Beverly MA; Cat. No. 95048). Quantitative reverse transcription-PCR was performed using PerfectA SYBR Green 
(Quantabio, Beverly MA; Cat. No. 95072) with $100 \mu \mathrm{g}$ of cDNA used per reaction. Primer sequences are listed in Table I. Cycle numbers were normalized to to $\beta$-actin and vehicle-treated or empty vector cells and scaled to $1, n=3$. For patient-derived xenografts, explants were collected after 72 hours, and RNA was extracted using QIAzol Lysis Reagent (Cat No. 79306; Qiagen, Valencia, CA, USA) and dissection of the tumor with scissors and then RNA was extracted using the Quick RNA Mini Prep Kit (Zymo Research, Irvine, CA).

\section{Fluorescence staining for cell morphology and proliferation}

Cells were fixed in formalin ( $10 \%$ buffered formalin phosphate, Fischer Scientific, Hampton $\mathrm{NH}$ ) and permeabilized with Triton-X100 (MP Biomedicals, St. Ana CA). Cytoskeletal components were stained with AlexaFluor 555-conjugated phalloidin (Cell Signaling, clone 8953, 1:200, Danvers MA). For proliferation studies, cells were stained with a primary conjugated antibody against Ki-67 (BD Biosciences; 1:200). Cells were counterstained with DAPI (NucBlue Fixed Cell Stain ReadyProbe, Life Technologies, Carlsbad CA). ApoTome (commercial structure illumination microscopy by Zeiss, Thornwood, NY) fluorescent images were captured on an inverted microscope (Zeiss) and digitally filtered to obtain optical slices. For Ki-67 staining, quantified results are represented as percent positive Ki-67 staining (red) of total number of cells visualized by DAPI nuclear stain (blue).

\section{In vivo xenograft experiments}

Immunocompromised SCID/Beige mice (4-6 weeks of age), described previously, acclimated for 5 days before initiation of experiments. Mice ( 5 mice per group) were inoculated with cells grown in tissue culture, $2.5 \times 10^{6}$ cells per injection in the TU-BcX-4IC-shNEK5 and MDA-MB-231-shNEK5 experiments and $5 \times 10^{6}$ cells per injection in the MCF7 xenograft experiment. Each mouse inoculated with MCF-7 cells were ovariectomized and implanted with a $17 \beta$-estradiol tablet in the back subcutaneously using a trocar. Cells were mixed in a 2:1 ratio with Matrige ${ }^{\mathrm{TM}}$ (Corning, Corning NY; Cat. No. CB-40234) and PBS. For the shRNA mouse experiments, in the '+Dox' group mice were fed $200 \mathrm{mg} / \mathrm{kg}$ doxycycline chow (Bio-Serv, Flemington NJ; Cat. No. S3888). Tumors were measured biweekly with calipers and tumor volume was calculated. After tumors reached $850 \mathrm{~mm}^{3}$ in volume tumors were resected. 20 days after survival surgery, mice were euthanized, and lungs and livers were harvested. Lungs and livers were formalin fixed, paraffin-embedded, sectioned and stained with hematoxylin and eosin ( $\mathrm{H} \& \mathrm{E})$ to visualize metastatic lesions. Number and areas of visualized metastases per section were quantified using ImageScope and recorded. Resected tumors were flash frozen and preserved at $-80^{\circ} \mathrm{C}$ for qPCR analyses.

\section{Microscopy Imaging}

The Nikon eclipse TE2000-s inverted fluorescence microscope and camera with x-cite series 120 illuminator (Nikon; Melville, NY), in conjunction with IP Lab version 3.7 software (Rockville, MD) were used in the visualization of crystal violet-treated and phalloidin stained cells to observe morphological changes.

\section{Hematoxylin \& Eosin staining}


Livers, lungs and tumor tissues were processed by the Department of Histology and Pathology at Tulane University. As per standard protocol, formalin-fixed tissues were paraffin-embedded and sectioned at 4 $\mu \mathrm{M}$ and mounted on glass slides. Mounted sections were then exposed to xylene, ethanol, and acetic acid with intermittent washings with water before being stained with hematoxylin and eosin ( $H \& E$ ). After staining, slides were then again exposed to ethanol and xylene to complete the protocol.

\section{Statistical analysis}

Studies run in triplicate were analyzed by unpaired Student's $t$ test (Graph Pad Prism V.4). $p$-Values $<0.05$ were considered statistically significant.

\section{Results}

\section{NEK5 baseline expression in patient-derived breast tumors and xenografts}

To assess NEK5 expression at baseline in breast tumors, we assessed NEK5 protein expression in breast tumors. Cell-line derived xenografts (MCF-7, ER + subtype; MDA-MB-231, TNBC) and patient-derived tumors propagated in mice were analyzed. Every PDX model utilized in this study represented patients with TNBC tumors in the greater New Orleans area. TU-BcX-4IC represented a highly aggressive metaplastic breast carcinoma, TNBC subtype. Two of the models were derived from metastatic sites, with TU-BCX-4EA-LNb4 derived from a lymph node and TU-BcX-49S derived from a liver metastatic lesion. One model, TU-BcX-4M4, generated two PDX models from two distinct sections of a mastectomy specimen, $\mathrm{Ta}$ and Tb. The metastatic profiles of the PDX models utilized in the patients were as follows: TU-BcX-4IC and TU-BcX-4QX represented tumors that were chemo-refractory and very invasive, with rapid growth rates and high rates of metastases. TU-BcX-2K1 was derived from the biopsy of a treatment-naïve tumor that was lowly metastatic; TU-BcX-4EK represented a non-metastatic tumor resistant to chemotherapy. TU-BcX-4M4Tb was a lowly metastatic tumor that was chemo-refractory. NEK5 protein expression was examined in the paraffin embedded and sectioned tumors using immunohistochemistry with a NEK5specific antibody; mouse testis tissue was used as the positive control. NEK5 expression was strongly expressed in MDA-MB-231 tumors compared to the ER + xenografts from MCF-7 cells (Fig. 1). In the PDX tissue, there was more abundant expression in TU-BcX-4M4Ta/Tb and - 4QX tumors, less abundant expression in TU-BcX-4EA-LN, -4IC and - 4QX tumors, and absent expression in the TU-BcX-49S PDX model. Interestingly, NEK5 expression was nuclear in 4IC and 4EA-LN tumors but is localized to the cell membranes of $4 \mathrm{M} 4$ tumors. With respect to localization of NEK5 expression, we are the first to demonstrate a difference in distribution of NEK5 expression in individual patient tumors. Whether this pattern correlates with tumor behavior, or if it is reflective of differences in NEK5 localization depending on unique inherent features of individual breast tumors remains unexplored. The apparent differences in NEK5 expression amongst individual patient tumors, with no reflection on metastatic potential or subtype (all PDX models represented TNBC tumors), suggests NEK5 expression correlates with unique molecular or histological features of individual patient tumors. 
Next, NEK5 baseline gene expression was evaluated using qPCR of various cell lines. Established cell lines were employed representing ER + breast cancer subtypes (MCF-7, BT-20) and TNBC (Hs-578T, MDAMB-157, BT-549, MDA-MB-231) and primary cells derived from TNBC tumors (TU-BcX-2K1, TU-BcX-2O0, TU-BCX-4IC). While NEK5 expression was lower in the ER + cells compared to TNBC lines, within TNBC cells NEK5 varied amongst the individual cell lines tested (Supplementary Figure S1). Together, these protein and genomic analyses in various tumors and cell lines demonstrate NEK5 baseline expression is not uniform amongst individual patient's tumors and does not follow subtype-specific patterns in breast cancer. These conclusions were especially true in TNBC subtypes, and there were no patterns in NEK5 expression amongst the established cell line-derived xenografts based on current categorization of TNBC based on molecular characteristics $[4,5]$.

\section{Overexpression of NEK5 alters the epithelial-like cell morphology and promotes a migratory phenotype}

To test the function of NEK5 in breast cancers, we used a luminal A ER + cell line, MCF7, that at baseline exhibits an epithelial-like morphology (rounder cells, increased cell-cell contacts, cobblestone appearance) and is lowly metastatic. MCF-7 cells were stably transfected with NEK5 or empty vector constructs as the control; neomycin was used as the selection agent and clones were generated. NEK5 overexpression was confirmed on a transcript level with qPCR (Fig. 2A) and on a protein level with Western blot (Fig. 2B). Effects of NEK5 overexpression on cell morphology was then evaluated using crystal violet staining and phalloidin fluorescence staining. We observed overall increases in the areas of individual cells with larger volumes of cytoplasm and fewer cell-cell contacts in NEK5 overexpressing cells compared to vector cells (Fig. 2C; Supplementary Figure S2), representative of changes in the cytoskeletal structure of the epithelial-like MCF-7 cells. The effect of NEK5 overexpression on cell migration was evaluated using a transwell migration assay, where cell culture media was the chemoattractant and cells were seeded in the upper chamber in growth factor-reduced media. MCF-7 cells exhibit low migratory capacity at baseline; compared to MCF7-VEC cells, NEK5 overexpression significantly increased cell migration (Fig. 2D, Supplementary Figure S3). The NEK family of kinases regulate multiple processes integral in proper cell cycle progression [21, 22]. We then assessed cell proliferative effects of NEK5 overexpression in the MCF-7 cells using Ki-67 staining and fluorescence imaging [29]. Based on representative images, we observed a decrease in Ki-67 signal in NEK5overexpressing cells (Fig. 2E). Furthermore, quantification revealed a significant reduction in the number of proliferating cells (Fig. 2F).

Knockdown of NEK5 using TET-on shRNA system demonstrated a suppression of TNBC cell migration independent of cell proliferation

After demonstrating that forced overexpression of NEK5 is sufficient to drive migration in a cell line with low migratory potential at baseline, we then sought to explore effects of NEK5 knockdown on these processes. For these experiments we employed a TET-on doxycycline inducible shRNA system and stably transfected TNBC cells that exhibited mesenchymal and migratory phenotypes at baseline. NEK5 
expression was knocked down in two TNBC cell lines: MDA-MB-231 and a patient-derived primary cell line established in our laboratory, TU-BCX-4IC. Cells were stably transfected with shNEK5 or shLT3GPIR empty vector control, selected with puromycin, and clones were generated. To obtain NEK5 knockdown, doxycycline $(2 \mu \mathrm{g} / \mathrm{mL})$ was added to the cell culture media, and results were compared to plated cells in the absence of doxycycline (the experimental control). NEK5 knockdown was confirmed on a transcript level with qPCR (Fig. 4A) and on a protein level with Western blot (Fig. 4B, C).

Cell migration was again assessed using transwell migration assays. DMEM media containing $10 \%$ FBS was used as a chemoattractant in the bottom chamber and MDA-MB-231-shNEK5 or TU-BcX-4IC-shNEK5 cells pre-treated for 48 hours with doxycycline to induce NEK5 knockdown (or untreated negative controls) were plated in the top chamber with low growth serum-containing media. NEK5 knockdown in both MDA-MB-231 and TU-BCX-4IC cells significantly suppressed cell migration of invasive TNBC cells (Fig. 5A, B). While NEK5 knockdown did not appear to dramatically affect cell morphology on crystal violet staining (Supplementary Figure S4), we used phalloidin fluorescence staining to highlight actin filaments and further investigate effects of NEK5 knockdown on cell morphology of TNBC cells (Fig. 5C). Subsequent morphologic analysis and quantification of cell parameters including area, perimeter, circularity and nuclear:cytoplasm area ratios were then assessed. Morphometric quantification revealed NEK5 knockdown significantly altered cell morphology parameters, reverting the classic mesenchymal TNBC morphology (cellular protrusions, decreased cell-cell contact, no formation of colonies) to a more epithelial phenotype in TU-BcX-4IC cells but to a less degree with MDA-MB-231 cells (Supplementary Figure S5). Effects of NEK5 knockdown on supporting a more epithelial phenotype was characterized by rounder cells, increased cell-cell contact, and fewer cellular protrusions. Together with the data obtained using the MCF-7 luminal A cell line, these data demonstrate the NEK5 effects on cytoskeletal organization and cell morphology of breast cancer cells were cell type specific. Cell proliferation was then evaluated in the NEK5 knockdown cell lines using fluorescence staining to visualize Ki-67 positive cells. In MDA-MB231-shNEK5 cells, a significant reduction in the number of proliferating cells was observed compared to total (or DAPI-positive) cells. NEK5 knockdown in TU-BcX-4IC cells did not affect cell proliferation (Fig. 5D, E). These data, together with our findings that NEK5 overexpression did not increase cell proliferation, demonstrated that the effects of NEK5 on cell proliferation in TNBC may be cell line specific.

\section{NEK5 knockdown did not affect tumorigenesis and had cell line specific effects on metastasis}

We then sought to investigate in vivo effects of NEK5 activity on tumor growth kinetics and metastasis of breast cancer cells. Immunocompromised SCID/Beige mice were first inoculated with MCF7-NEK5 or -VEC cells coated in Matrige ${ }^{\text {TM }}$. At baseline, MCF-7 cells exhibit very low metastatic capabilities [30]. Tumor growth kinetics were similar between both groups, with no significant change in final tumor volume (850 $\mathrm{mm}^{3}$ ) after 21 days (Fig. 3A). At this time point, survival surgery was performed, and tumors were resected, to allow circulating tumor cells to seed and differences in metastases to be quantified. After 20 additional days, mice were sacrificed, and lungs and livers were harvested. Organs were paraffin- 
embedded, sectioned and H\&E stained to visualize metastases. Quantification of metastases revealed NEK5 overexpression increased the number of metastatic lesions in the lungs and livers (Fig. 3B-E). However, overall areas of the lesions were not significantly different between the two groups (Fig. 3F, G). These data suggest a role for NEK5 in acquisition of a migratory and metastatic phenotype independent of cell proliferation and tumor growth kinetics.

Similar in vivo experiments were performed with NEK5 knockdown cell lines. SCID/Beige mice were inoculated with MDA-MB-231-shNEK5 or TU-BcX-4IC-shNEK5 cells. Mice in the '-Dox' negative control group were fed normal chow, while mice in the '+Dox' group were fed doxycycline chow to induce NEK5 knockdown. There was no change in tumorigenesis in the NEK5 knockdown group compared to the negative controls in neither the MDA-MB-231-shNEK5 (Fig. 6A) nor in the TU-BcX-4IC-shNEK5 experiments (Fig. 6B). Lungs and livers were harvested after 20 days, formalin fixed, paraffin embedded, and $\mathrm{H}$ \& $\mathrm{E}$ stained. NEK5 knockdown did not affect lung metastases in MDA-MB-231-shNEK5 (Fig. 6C-E) or TU-BcX4IC-shNEK5 (Fig. 6F-H) xenografts in -Dox groups compared to + Dox groups. NEK5 knockdown reduced average liver metastatic lesion areas in both MDA-MB-231 (Fig. 6I, J) and TU-BcX-4IC xenografts (Fig. 6J, M). NEK5 knockdown also reduced the total number of liver metastases per section in both MDA-MB-231 (Fig. 6L) and TU-BcX-4IC experiments (Fig. 6N). However, only the data with quantified number of liver metastases in TU-BcX-4IC-shNEK5 xenografts in -Dox compared to + Dox groups was significantly reduced. These data showed that NEK5 knockdown suppressed liver metastasis, but not lung metastases. After euthanasia, cells were harvested from the peritoneal fluid of both groups to isolate metastatic cells. To further evaluate the effects of NEK5 knockdown on metastasis, the in vivo effect of NEK5 knockdown on cell proliferation was evaluated using a colony assay. Cells were plated in a 6-well plate (1,000 cells per well) to evaluate proliferation (Supplementary Figure S6A, B). There was an overall decrease in number of colonies formed in the + Dox group compared to the -Dox group, showing that NEK5 knockdown suppressed colony forming capabilities of metastatic cells (Supplementary Figure S6C, D). These data, together with the observation that NEK5 activity did not affect tumor growth kinetics and the finding that NEK5 activity altered the number of metastases, but not the overall areas, further supports a role for NEK5 in regulation of breast cancer cells' capabilities to escape from the primary tumor but does not affect seeding nor colonization capabilities once at the distal tissue site.

\section{Discussion}

Kinase-targeting small molecule inhibitors have transformed the field of therapeutic discovery in diverse tumor types. Mitotic kinases are commonly targeted in cancer because they broadly disrupt cell cycle regulation but exhibit cytotoxic adverse events in early clinical studies $[17,18,19]$. NEK5 is the least studied member in the NEK family of mitotic kinases. While NEK5 remains widely understudied in the context of cancer, as previously mentioned there is an emerging focus on characterizing roles of individual NEK family members in various oncologic and pathologic processes. Specifically, groups have demonstrated NEK5 activity in centrosome disjunction, myogenesis, cell cycle progression, caspase-3 activity, and regulation of mitochondrial functions. Most recently Ferezin et al. revealed NEK5 regulates mitochondrial homeostasis and mtDNA maintenance due to potential interactions with mitochondrial 
proteins [31]. The emergence of more publications with respect to characterizing NEK5 activity demonstrate that the scientific community recognizes potentially important roles of NEK5 in regulating key biologic processes [21]. In fact, NEK5 has been identified as a kinase of interest in sequencing and phenotypic screens in other cancer types [32].

In cancer systems, recent studies have focused on characterizing a biological role for NEK5 in prostate and thyroid cancers. In thyroid malignancies, Melo-Hanchuk et al. demonstrated that NEK5 expression was higher in patients with invasive and metastatic tumors, and in patients presenting with larger tumor volumes, suggesting a role for NEK5 expression in more clinically aggressive tumors [18]. Specifically, in the context of breast cancer many biologic processes that NEK5 activity has been associated with also have significant functions in the pathophysiology of cancer development. NEK5 regulates centrosome integrity in cell cycle processes $[20,21,25]$ and mediates other cell intrinsic pathways involved in cell proliferation and migration [27]. In addition to other NEK family members, NEK5 activity has been associated with the DNA damage response and regulation of genomic stability [33]. Melo Hanchuk et al. have shown that NEK5 is associated with the DNA damage response through regulation of topoisomerase Ilb activity [26]. Furthermore, a feature that makes NEK5 structurally unique compared to other NEK members is the presence of a DEAD box domain; potential contributions of this domain to NEK5 function is not yet known. In breast cancer systems, Pei et al. described a role for NEK5 in cell proliferation through regulation of Cyclin A2 expression and demonstrated NEK5 prevented cell apoptosis and increased S phase of breast cancer cells [27]. In the TNBC cells MDA-MB-231 and MDA-MB-468 cells they demonstrated that NEK5 promoted migration and invasion of breast cancer cells [27].

This project further characterized roles for NEK5 in breast cancer; we employed derivations of PDX model systems representing patients in the greater New Orleans area to provide more translational evidence for our findings. Similar to the Pei et al. study utilizing TNBC cells, we also showed that NEK5 expression drove cell migration of breast cancer cells representing different breast cancer subtypes including triple negative (MDA-MB-231, TU-BcX-4IC) and luminal A (MCF-7). Together these findings demonstrate NEK5 drives a migratory cell phenotype in a non-subtype dependent manner.

The immunohistochemistry experiments revealed NEK5 expression is cell type specific in breast cancer and does not follow patterns based on conventional categorization of breast cancer subtypes based on receptor status. It is important to note that we did not molecularly characterize the PDX tumors tested using transcriptomic sequencing, which may reveal patterns of NEK5 expression using a more recent system to categorize breast cancers based on molecular features $[4,5]$. However, it is also notable that NEK5 expression was present in all but one of the PDX tumors tested, with variable expression in individual tumors. The more aggressive PDX tumors with higher growth rates in murine models and in patients, TU-BcX-4QX and TU-BcX-4IC, exhibited moderate levels of NEK5 expression. Interestingly, NEK5 expression varied dramatically in the PDX models that were established from different locations of the same mastectomy specimen, TU-BcX-4M4Ta and TU-BcX-4M4Tb. These observations demonstrate that not only is NEK5 expression not homogeneous in patient breast tumors, but it also presents the hypothesis that specific populations with higher NEK5 expression may be the subclonal cell populations 
responsible for driving a more migratory cell phenotype. More extensive studies characterizing these observations are required before reporting more concrete conclusions for this hypothesis.

An interesting observation from our study was that while NEK5 activity regulated cell migration and cytoskeletal structure in vitro, it did not affect cell proliferation as determined by $\mathrm{Ki}-67$ staining. We did not observe consistent data with respect to cell proliferation (as quantified by Ki-67 staining) and NEK5 activity. Together with data from Pei et. al, these data support that the proliferative effects of NEK5 are cell type specific and the effects of NEK5 on cell migration and cytoskeletal changes are independent of proliferation effects. These observations, compounded with the data that NEK5 activity affected the number of metastases to distal tissue sites, but did not affect the overall area of these metastases, demonstrated that NEK5 can affect seeding capabilities of cells but did not alter cell proliferation capabilities after seeding. In other words, while NEK5 may regulate the initiation step of metastasis, it may have lesser impact on metastatic cell colonization and proliferation. Our data and interpretation are consistent with prior studies that demonstrated NEK5 activity on organization of cytoskeletal components, including microtubules, a process that has important implications in cell migration and metastasis. Mechanistically, NEK5 has been shown to interact with NEK4 and NEK6 to regulate microtubule organization in epidermal cells [25], suggesting a role for NEK5 in metastatic progression possibly through reorganization of cytoskeletal components. We utilized a knockdown approach to examine NEK5 activity in vivo. Given the observation that shRNA knockdown was not sufficient to fully suppress metastasis, follow-up experiments will utilize CRISPR/Cas9-mediated knockout of NEK5 to evaluate if complete loss of NEK5 expression is necessary to inhibit the metastatic phenotype.

The purpose of this project was to characterize NEK5 function in breast cancer biology, a severely understudied kinase that has recently been a focus of investigation of having crucial regulatory functions in cancer biology. We propose NEK5 regulation of cytoskeletal components may regulate the observed migratory phenotype in NEK5-activated cells and the underlying mechanisms behind this behavior will be explored in the future mechanism-driven studies.

\section{Conclusion}

Mitotic kinases are attractive targets in breast cancer, due to direct cell cycle regulatory functions in cancer systems. NEK5, a member of the NIMA-Related kinase family is understudied in breast cancer. Our findings support a role for NEK5 in regulation of the migratory/metastatic axis in breast cancer independent of its effects on cell proliferation and tumor growth kinetics, and we identified potential downstream targets and signaling pathways to pursue.

\section{Declarations}

\section{Ethics approval:}


Experiments performed in this study comply with $\mathrm{NIH}$ regulations and all applicable international, national, and/or institutional guidelines for the care and use of animals were followed. All procedures performed in studies involving animals were in accordance with the ethical standards of the Institutional Animal Care and Use Committee at Tulane University.

\section{Availability of data and materials.}

All data generated or analyzed during this study are included in this published article and its additional information files.

\section{Competing Interests.}

The authors have no competing interests to declare.

\section{Funding.}

This project was funded in part by grant 1R03TR003386-01 from the NIH National Center for Advancing Translational Science (NCATS) as part of the Illuminating the Druggable Genome (IDG) Project. This work was also supported by grants from National Institute of Health $(\mathrm{NIH})$ (Grants No. R01-CA174785-A1 (BCB) and R01CA125806-02 (MEB)).

\section{Authors' Contributions.}

MDM wrote the first draft of the manuscript and contributed to project conception and experimental design, and data acquisition, analysis and interpretation. SE, VTH, HEB, MKW, MA, TY, TC, HW, GW contributed to data acquisition, analysis and interpretation and provided edits to the manuscript text. WJZ, DHD, CW, NK contributed to project conception and experimental design as well as data analysis and interpretation. $A B, F F$ and KPN provided the RNA sequencing data acquisition and analysis. BMC-B and MEB provided the necessary funds and materials to complete this project, contributed to project conception and experimental design, and revised the manuscript.

Consent for publication: Not applicable.

\section{Acknowledgements:}

We acknowledge Histology Core facilities at Tulane University for the hematoxylin and eosin stains of the tissue. We acknowledge the support and expertise of the University of Carolina Eshelman School of Pharmacy and their extensive contributions to this project. The SGC is a registered charity (number 1097737) that receives funds from AbbVie, Bayer Pharma AG, Boehringer Ingelheim, Canada Foundation for Innovation, Eshelman Institute for Innovation, Genome Canada, Innovative Medicines Initiative (EU/EFPIA), Janssen, Merck KGaA Darmstadt Germany, MSD, Novartis Pharma AG, Ontario Ministry of Economic Development and Innovation, Pfizer, Takeda, and Wellcome [106169/ZZ14/Z]. Additionally, we are always grateful for the support of the Krewe de Pink organization in New Orleans and importantly, we 
thank the patients who donated their breast cancer tissue specimens to benefit breast cancer research; their contributions are valued and appreciated.

\section{References}

1. DeSantis CE, Ma J, Gaudet MM, Newman LA, Miller KD, Sauer AG et al. Breast cancer statistics, 2019; CA: A Cancer Journal for Clinicians. 2019;69(6). DOI:10.3322/caac.21583

2. Bianchini G, Balko JM, Mayer IA, Sanders ME, Gianni L (2016) Triple-negative breast cancer: challenges and opportunities of a heterogeneous disease. Nat Rev Clin Oncol 13:674-690

3. Metzger-Filho O, Tutt A, de Azambuja E, Saini KS, Viale G, Loi S et al (2012) Dissecting the heterogeneity of triple-negative breast cancer. J Clin Oncol 30(15):1879-1887

4. Lehmann BJ, Bauer J, Chen X, Sanders ME, Chakravarthy BA, Shyr Y et al (2011) Identification of human triple-negative breast cancer subtypes and preclinical models for selection of targeted therapies. J Clin Invest 121:2750-2767

5. Lehmann BD, Jovanovic B, Chen X, Estrada KN, Johnson KN, Shyr Y et al (2016) Refinement of triplenegative breast cancer molecular subtypes: implications for neoadjuvant chemotherapy selection. PLOS ONE. DOI:10.1371/journal.pone.0157368

6. Kennecke H, Yerushalmi R, Woods R, Cheang MC, Voduc D, Speers CH et al (2010) Metastatic behavior of breast cancer subtypes. J Clin Oncol 28:3271-3277

7. Gerratana L, Fanotto V, Bonotto M, Bolzonello S, Minisini AM, Fasola G et al (2015) Pattern of metastasis and outcome in patients with breast cancer. Clin Exp Metastasis 32:125-133

8. Lyden D, Welch DR, Psaila B. Cancer metastasis: biologic basis and therapeutics. Cambridge University Press; 2011

9. Neophytou C, Boutsikos P, Papageorgis P (2018) Molecular mechanisms and emerging therapeutic targets of triple-negative breast cancer metastasis. Front Oncol. DOI:10.3389/fonc.2018.00031

10. Lee A, Djamgoz MBA (2018) Triple negative breast cancer: Emerging therapeutic modalities and novel combination therapies. Cancer Treat Rev 62:110-122

11. Nagini S (2017) Breast cancer: Current molecular therapeutic targets and new players. Anticancer Agents Med Chem 17(2):152-163

12. Bhullar KS, Lagaron NO, McGowan EM, Parmar I, Jha A, Hubbard BP, Rupasinghe HPV. Kinasetargeted cancer therapies: progress, challenges and future directions. Molecular Cancer. 2018;17(48):DOI:10.1186/s12943-018-0804-2

13. Midland AA, Whittle MC, Duncan JS, Abell AN, Nakamura K, Zawistowski JS et al (2012) Defining the expressed breast cancer kinome. Cell Res 22:620-623

14. Carles F, Bourg S, Meyer C, Bonnet P (2018) PKIDB: A curated, annotated and updated database of protein kinase inhibitors in clinical trials. Molecules 23:908

15. Manning G, Whyte DB, Martinez R, Hunter T, Sudarsanam (2002) S. The protein kinase complement of the human genome. Science 298:1912-1934 
16. Oprea TI, Bologa CG, Brunak S, Campbell A, Gan GN, Gaulton A et al (2018) Unexplored therapeutic opportunities in the human genome. Nat Rev: Drug Discovery 17:317-322

17. Welburn JPI, Jeyaprakash AA (2018) Mechanisms of mitotic kinase regulation: A structural perspective. Front Cell Dev Biol. DOI:10.3389/fcell.2018.00006

18. Bayliss R, Fry A, Hag T, Yeoh S (2012) On the molecular mechanisms of mitotic kinase activation. Open Biol 2(11):120136

19. de Carcer G, Perez de Castro I, Malumbres M (2007) Targeting cell cycle kinases for cancer therapy. Curr Med Chem 14(9):969-985

20. Fry AM, Bayliss R, Roig J (2017) Mitotic regulation by NEK kinase networks. Front Cell Dev Biol. DOI:10.3389/fcell.2017.00102

21. de Oliveira AP, Issayama LK, Pavan ICB, Silva FR, Melo-Hanchuk TD, Simabuco FM, Kobarg J (2020) Checking NEKs: Overcoming a bottleneck in human diseases. Molecules 25(8):1778

22. Prosser SL, Sahota NK, Peletier L, Morrison CG, Fry AM (2015) Nek5 promotes centrosome integrity in interphase and loss of centrosome cohesion in mitosis. J Cell Biol 209(3):339-348

23. Melo-Hanchuk TD, Martins MB, Cunha LL, Soares FA, Ward LS, Vassallo J, Kobarg J. Expression of the NEK family in normal and cancer tissue: an immunohistochemical study. BMC Cancer 2020;20(23). DOI:10.1186/s12885-019-6408-4

24. Thomas J, Morle L, Soulavie F, Laurencon A, Sagnol S, Durand B. Transcriptional control of genes involved in ciliogenesis: a first step in making cilia. Biol Cell. 2012;102(9). DOI:10.1042/BC20100035

25. Motose H, Hamada T, Yoshimoto K, Murata T, Hasebe M, Watanabe $Y$ et al (2011) NIMA-related kinases 6,4 , and 5 interact with each other to regulate microtubule organization during epidermal cell expansion in Arabidopsis thaliana. Plant J 67:993-1005

26. Melo-Hanchuk TD, Slepicka PF, Pelegrini AL, Menck CFM, Kobarg J (2019) NEK5 interacts with topoisomerase $\| \beta$ and is involved in the DNA damage response induced by etoposide. J Cell Biochem. DOI:10.1002/jcb.28943

27. Pei J, Zhang J, Yang X, Wu Z, Sun C, Wang Z, Wang B (2019) NEK5 promotes breast cancer cell proliferation through up-regulation of Cyclin A2. Mol Carcinog. DOI:10.1002/mc.22982

28. Chang TC, Matossian MD, Elliott S, Burks HE, Sabol RA, Ucar DA et al (2020) Evaluation of deacetylase inhibition in metaplastic breast carcinoma using multiple derivations of preclinical models of a new patient-derived tumor. PLOS ONE. DOI:

29. Scholzen T, Gerdes J (2000) The Ki-67 protein: from the known and the unknown. J Cell Physiol 182(3):311-322

30. Rhodes LV, Short SP, Neel NF, Salvo VA, Zhu Y, Elliott S et al (2011) Cytokine receptor CXCR4 mediates estrogen-independent tumorigenesis, metastasis, and resistance to endocrine therapy in human breast cancer [published correction appears in Cancer Res. May 1;71(9):3432]. Cancer Res. 2011;71(2):603-613 
31. Ferezin CC, Basei FL, Melo-Hanchuk TD et al (2021) NEK5 interacts with LonP1 and its kinase activity is essential for the regulation of mitochondrial functions and mtDNA maintenance. FEBS Open Bio 11(3):546-563. DOI:10.1002/2211-5463.13108

32. Matossian MD, Wells Cl, Zuercher WJ, Collins-Burow BM, Drewry DH, Burow ME (2021) Targeting never-in-mitosis-A related kinase 5 in cancer: A review". Current Med Chem 28:1.

DOI:10.2174/0929867328666210322101749

33. Pavan ICB, Peres de Oliveira A, Dias PRF, Basei FL, Issayama LK, Ferezin CdC et al (2021) On Broken $\mathrm{Ne}(\mathrm{c}) \mathrm{ks}$ and Broken DNA: The Role of Human NEKs in the DNA Damage Response. Cells 10(3):507

\section{Figures}
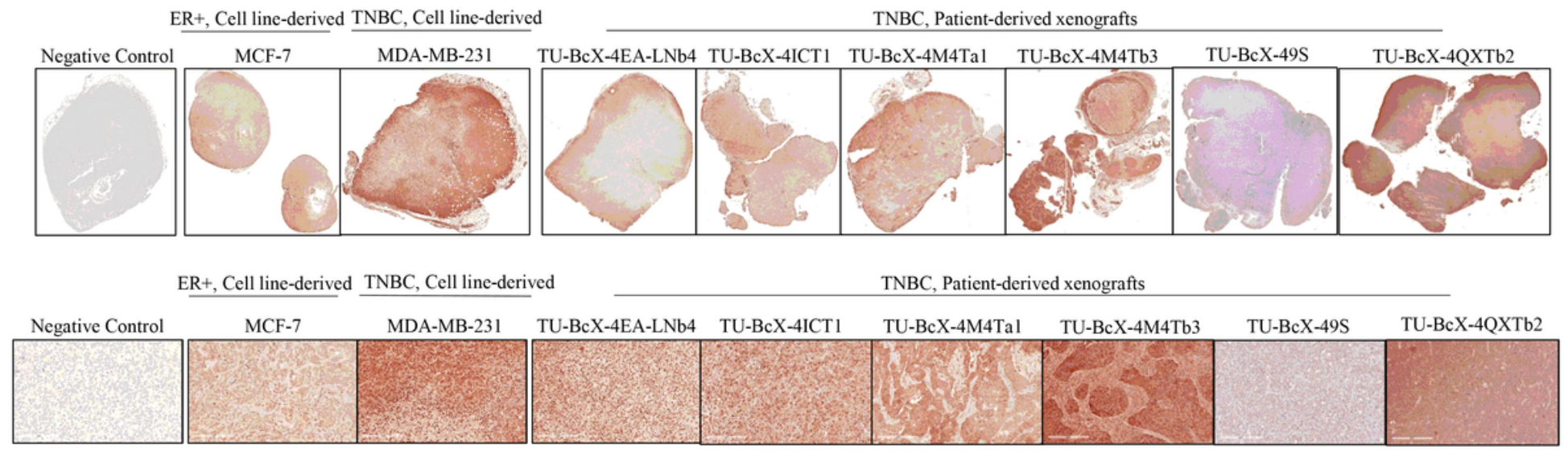

\section{Figure 1}

NEK5 protein expression in breast cancer tissue. Immunohistochemistry (IHC) staining of total NEK5 protein in PDXs and cell line-derived xenografts (MDA-MB-231, MCF-7 cells). PDX models employed are represented as 'TU-BCX-(model name)'. Further nomenclature of PDX models are as follows: ' $T$ ' represents the number of serial passages in mice (' $T 1$ ' represents one serial transplantation in a mouse), an ' $T a^{\prime}$ versus ' $T b$ ' represents two separate PDX models established from the same mastectomy specimen at different tissue sites. In addition to the 2K1, 4EK, 4M\$, 4QX and 4IC PDX tumors, 4EA-LN and 49S were evaluated. Mouse testis tissue known to have high endogenous NEK5 expression was used as positive controls. Representative images are shown at full scale and 200X magnifications. 


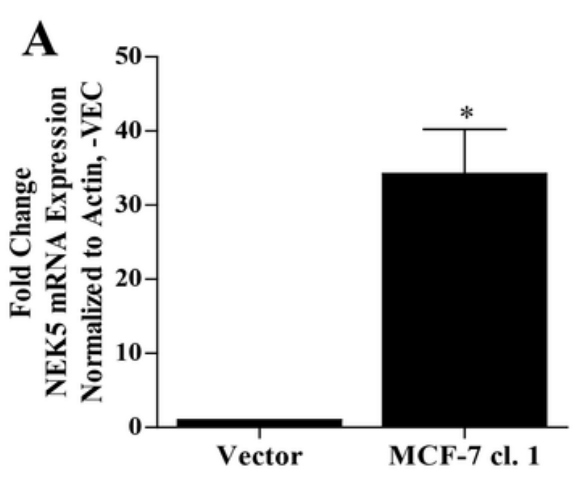

\section{B}

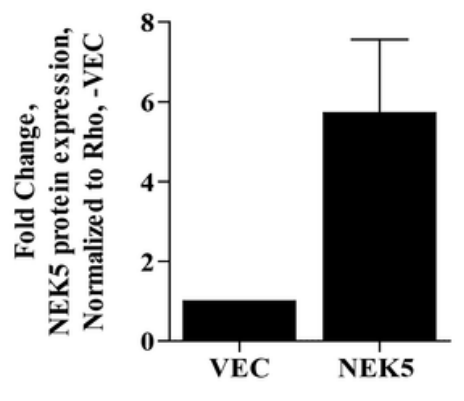

C

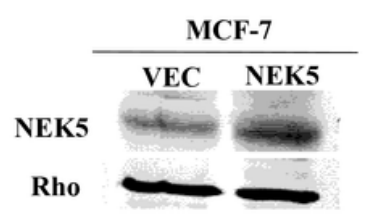

C

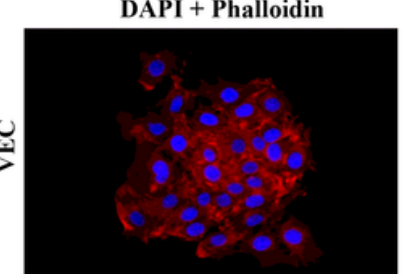

D
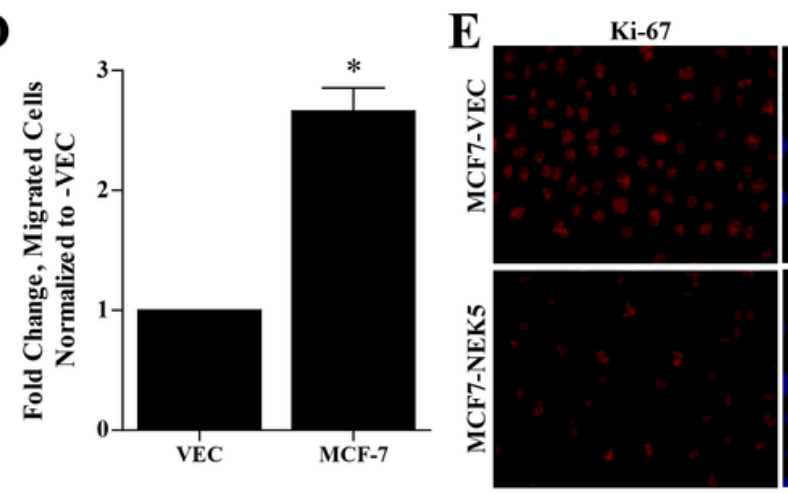

DAPI

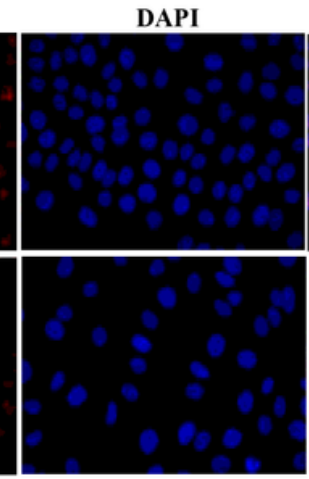

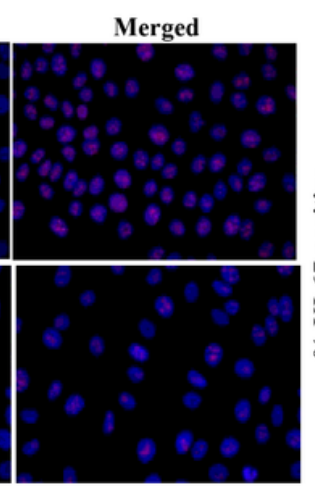
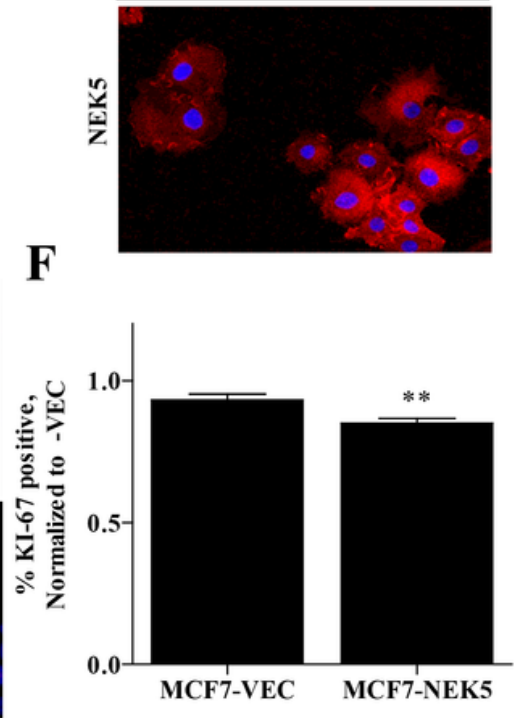

$\mathbf{F}$

Figure 2

NEK5 overexpression promoted cell migration but not proliferation in breast cancer cells. Stably transfected MCF7-NEK5 cells were confirmed for overexpression compared to cells transfected with empty vector controls. (A) MCF7-NEK5 mRNA overexpression was confirmed using qRT-PCR. Data was normalized to $\beta$-actin and vector controls and performed in triplicate. Error bars indicate S.E.M; ${ }^{*} p<0.05$. (B) MCF7-NEK5 protein overexpression was confirmed with Western blot. Data was normalized to Rho and empty vector controls and performed in duplicate. Error bars indicate S.E.M. MCF7-VEC or MCF7NEK5 cells were fixed with glutaraldehyde and stained. (C) Cell morphology was visualized fluorescence staining using phalloidin (red) to highlight actin filaments and DAPI nuclear stain (blue). IF images were captured using confocal microscopy, and crystal violet images were captures using brightfield microscopy. Transwell migration assay of MCF7-VEC and MCF7-NEK5 cells. DMEM containing $10 \%$ was used as the chemoattractant and the experiment was performed in triplicate. After 48 hours, membranes were removed, fixed in formalin and stained with crystal violet to visualize cells. (D) Quantification of average migrated cells per membrane using the ImageJ program. NEK5 overexpression significantly increased cell migration of non-invasive MCF7 cells. ${ }^{*} p<0.05$; error bars are shown as S.E.M. Cell proliferation was evaluated using an immunofluorescence stain with $\mathrm{Ki}-67$, a proliferation marker. (E) Red $=\mathrm{Ki}-67$, Blue = DAPI nuclear stain. Images were captured using confocal microscopy at 200X magnification. (F) Quantification of Ki-67 stain. Data is shown as percent Ki-67 positive cells compared to total, or DAPI-positive, cells. ${ }^{\star *} p<0.01$. 

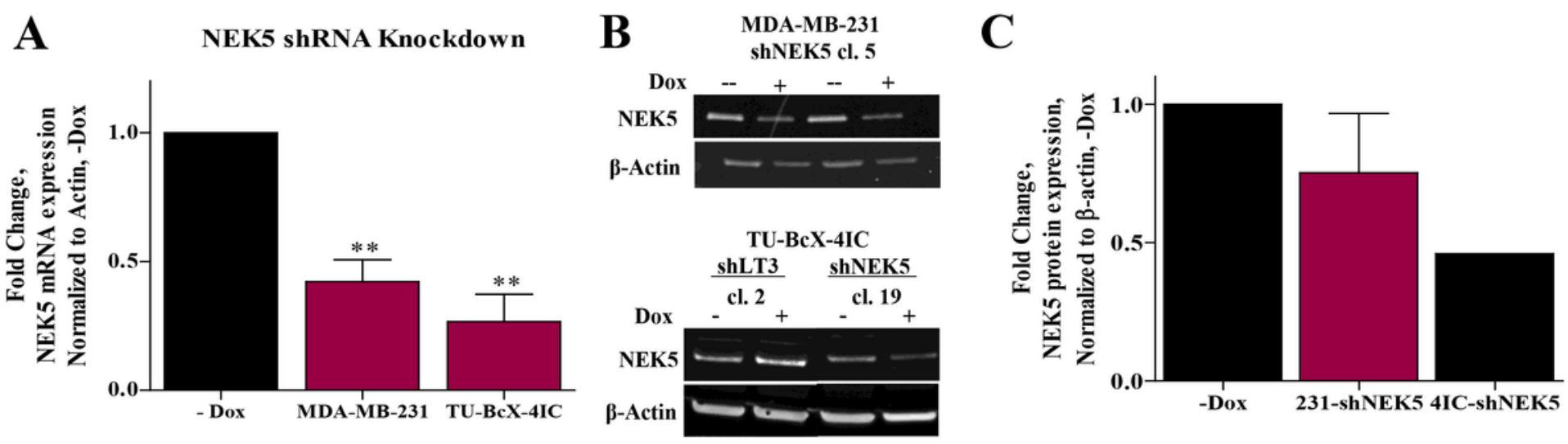

Figure 3

Doxycycline-inducible shRNA knockdown of NEK5 in TNBC cells. Cells were grown in the presence of doxycycline were compared to cells grown in the absence of doxycycline, denoted '-Dox'. (A) NEK5 knockdown was confirmed with qRT-PCR. Data was performed in triplicate and normalized to $\beta$-actin and -Dox and shown as fold change. ${ }^{\star \star} p<0.001$ and error bars are shown as S.E.M. (B, C) NEK5 knockdown was confirmed with Western blot; data was performed in duplicate and normalized to $\beta$-actin and -Dox and shown as fold change. Error bars are shown as S.E.M. 
A
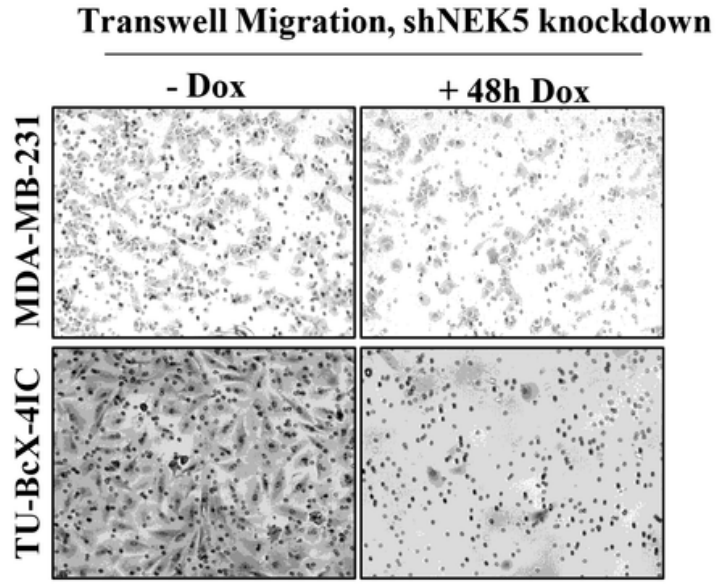

MDA-MB-231-shNEK5

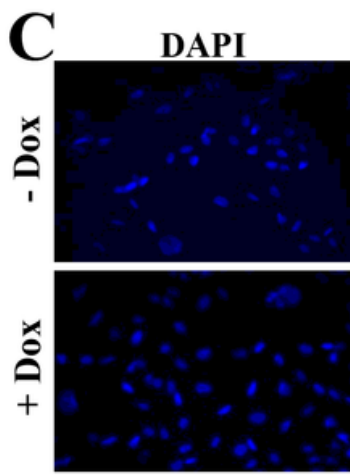

Phalloidin
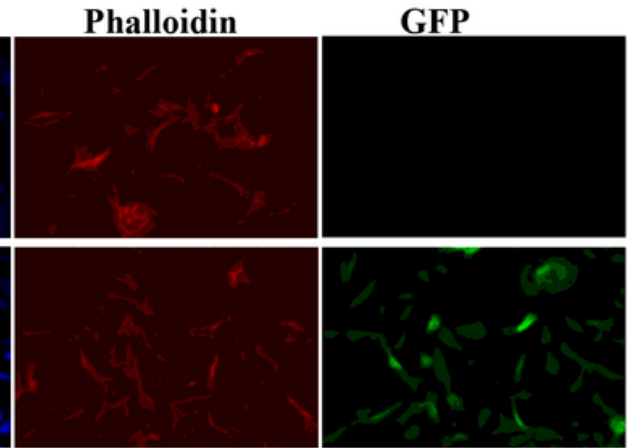

TU-BcX-4IC-shNEK5
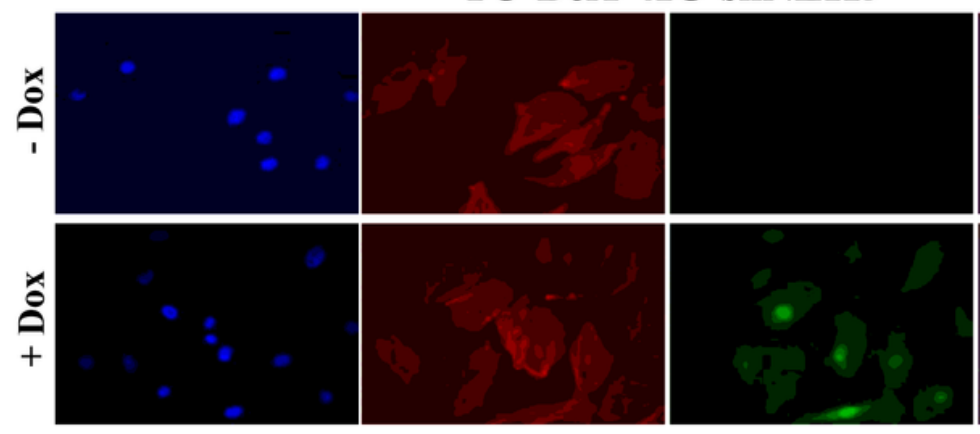

B

NEK5 shRNA Knockdown Migration

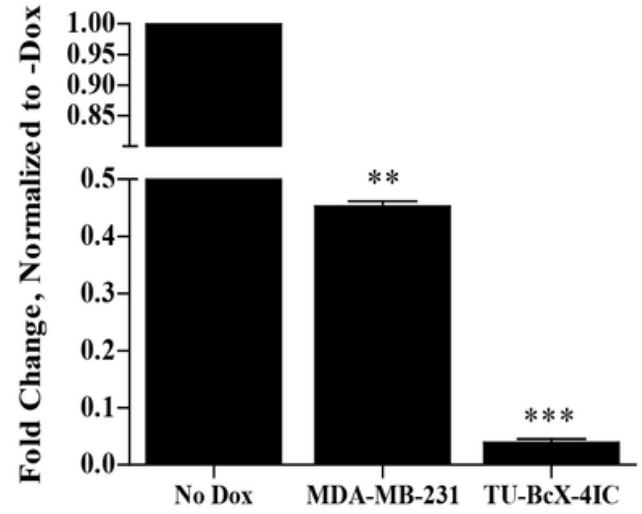

Merged
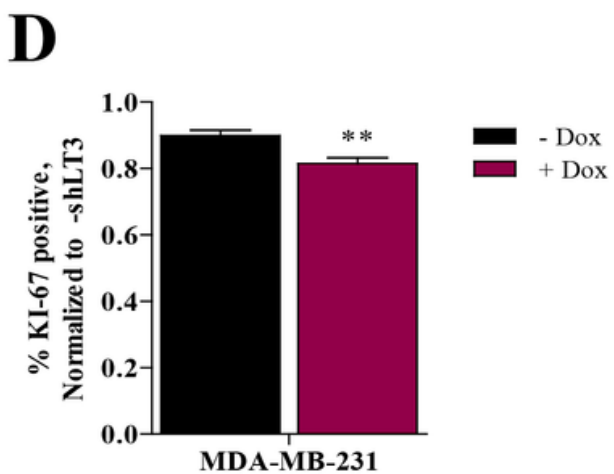

$\mathbf{E}$
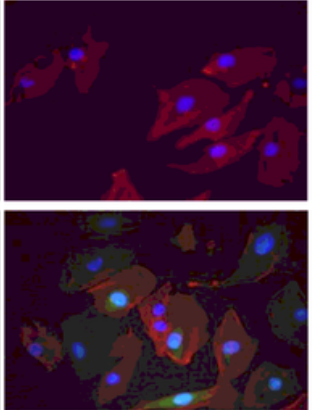

Figure 4

NEK5 knockdown suppressed cell migration in TNBC cells and suppresses proliferation in a cell type specific response. MDA-MB-231-shNEK5 and TU-BcX-4IC-shNEK5 cells were either treated with doxycycline for 48 hours at $2 \mu \mathrm{g} / \mathrm{mL}$ ('+Dox') to induce NEK5 knockdown, or untreated ('-Dox') as the negative control. (A) Cells (25,000 per assay) were seeded in the top layer of a transwell migration chamber suspended in low growth serum containing opti-MEM. DMEM containing 10\% FBS was placed in the bottom chamber as the chemoattractant, and cells migrated for 24 hours. Scrubbed membranes were formalin fixed and stained with crystal violet and imaged using brightfield microscopy. Representative images of membranes are shown. (B) Quantification of migrated cells was performed using the Image J program. Number of migrated cells was normalized to '-Dox' controls and fold change is represented. ** $p<0.01$, *** $p<0.001$; error bars are shown as S.E.M. MDA-MB-231-shNEK5 and TUBcX-4IC-shNEK5 cells were pre-treated for 48 hours with doxycycline ('+Dox') or in the absence of 
doxycycline ('-Dox'; control). Images were captured using confocal microscopy. (C) NEK5 knockdownmediated changes to cell morphologies were visualized with phalloidin cytoskeletal staining (red) and DAPI nuclear stain (blue). Cell proliferation was evaluated using fluorescence staining for Ki-67 (red) and DAPI nuclear stain (blue). Ki-67 positive cells were quantified in both (D) MDA-MB-231-shNEK5 and (E) TU-BcX-4IC-shNEK5 cells. Ki-67 positive cells were normalized to '-Dox' controls.

A

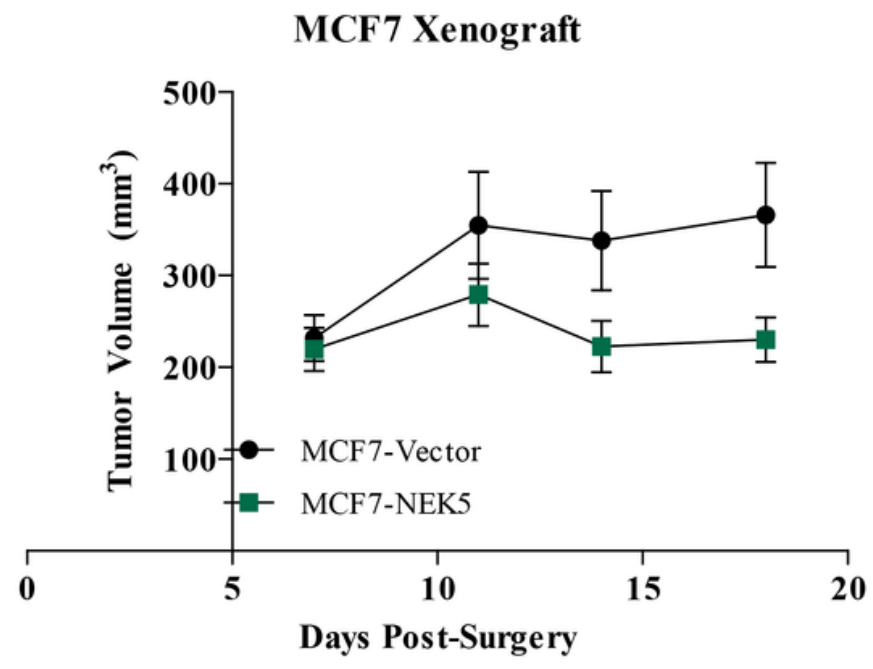

B

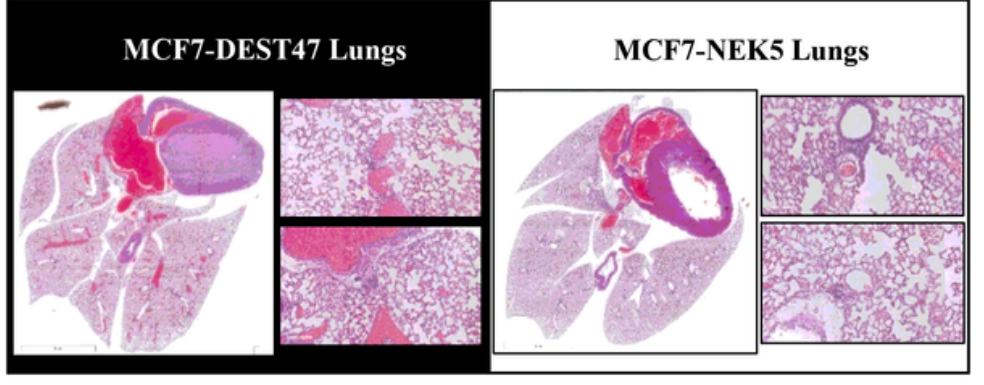

C
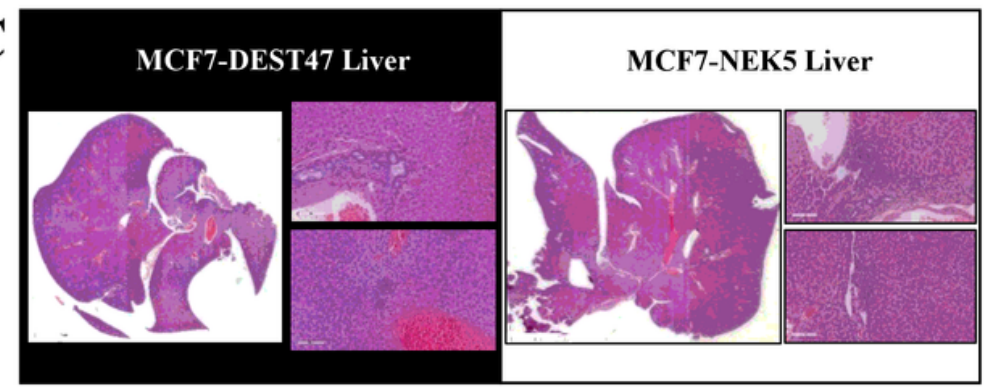

D MCF7 Xenografted Tumors Lung Metastases

E MCF7 Xenografted Tumors Liver Metastases

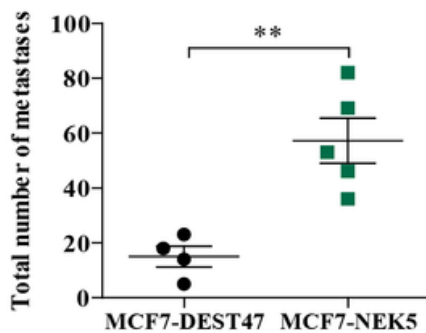

F MCF7 Xenografted Tumors Lung Metastases

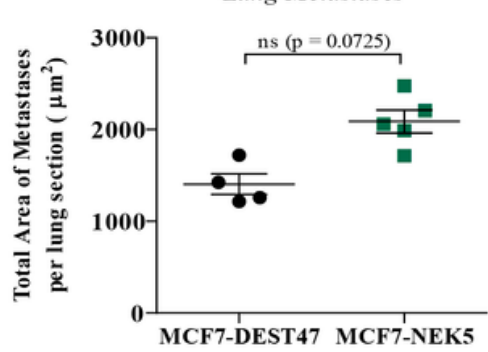

G MCF7 Xenografted Tumors

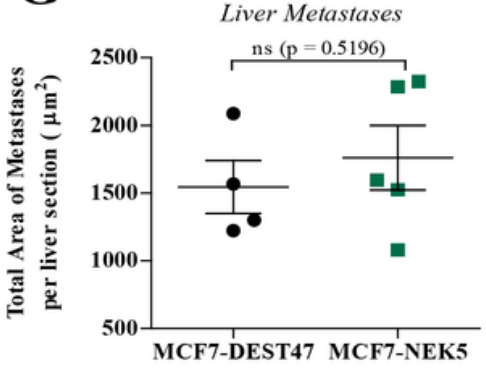

Figure 5

NEK5 overexpression did not affect tumor growth kinetics but increased metastatic potential in xenografted mice. Immunocompromised SCID/Beige mice were inoculated with MCF7-NEK5 or -VEC cells. (A) Tumor volumes of MCF7-VEC and MCF7-NEK5 xenografted cells. Error bars represent S.E.M. Survival surgery (tumor resection) was performed and after 20 additional days mice were euthanized. (B) Lungs and (C) livers were harvested, formalin-fixed, paraffin-embedded, sectioned and stained with $\mathrm{H}$ \& $\mathrm{E}$ to visualize and quantify metastasis. Inserts of representative images are shown at 200X magnification. Relative number of metastatic (D) lung and (E) liver lesions. Relative overall area of metastatic (F) lung and $(G)$ liver lesions. Data was quantified using the Aperio Image program and represented as the area $(\mu \mathrm{m} 3)$ of metastatic lesions per section. 

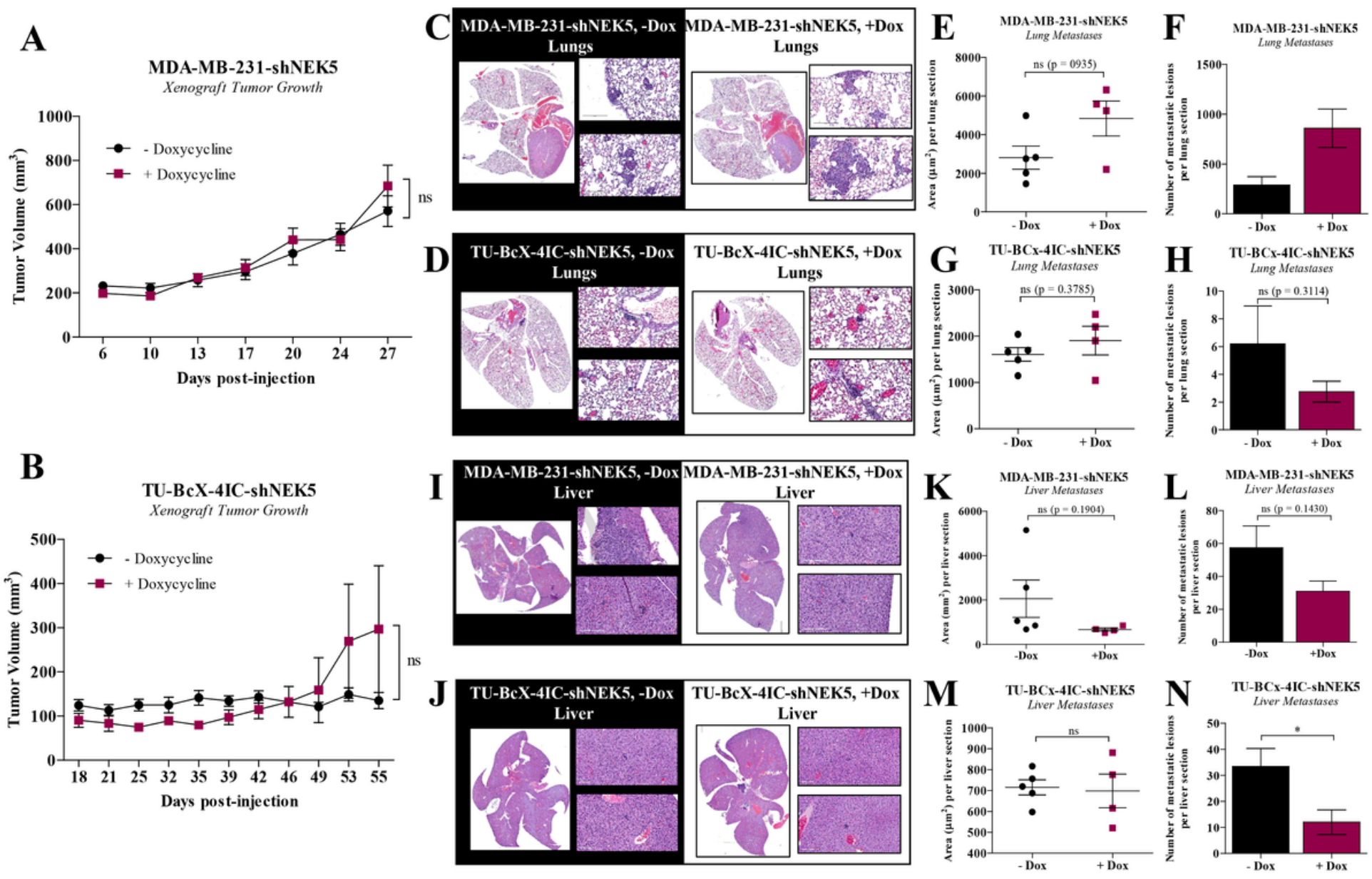

\section{Figure 6}

NEK5 knockdown suppressed metastatic potential of TNBC xenografts. SCID/Beige mice inoculated with MDA-MB-231-shNEK5 or TU-BcX-4IC-shNEK5 cells were either fed a normal diet ('-Dox' control group; $\mathrm{n}=5$ mice/group) or doxycycline chow ('+Dox' group; $n=5$ mice/group). Tumors were measured with calipers biweekly. Immunocompromised SCID/Beige mice were inoculated with MDA-MB-231-shNEK5 or TU-BcX4IC-shNEK5 cells and mice were fed doxycycline (+Dox) or normal chow (-Dox). Survival surgery (tumor resection) was performed and after 20 additional days mice were euthanized. Lungs were harvested, formalin-fixed, paraffin-embedded, sectioned and stained with $\mathrm{H} \& \mathrm{E}$ to visualize and quantify metastasis. Inserts of representative images are shown at 200X magnification for lungs from (A) MDA-MB-231 xenografts and (B) TU-BCX-4IC xenografts. Lungs and livers were harvested, formalin-fixed, paraffinembedded, sectioned and stained with $\mathrm{H} \& \mathrm{E}$ to visualize and quantify metastasis. Metastatic lesions were quantified using the Aperio Image program. Lung metastatic foci are represented as the $(F, H)$ area $(\mu \mathrm{m} 3)$ of lung metastatic foci per section and (D, F) relative number of metastatic lesions per lung section is shown between the -Dox and +Dox groups. Liver metastatic foci are represented as the $(K, M)$ area $(\mu \mathrm{m} 3)$ of metastatic lesions per section and $(L, N)$ relative number of metastatic lesions per lung section is shown between the -Dox and +Dox groups. 